\title{
Single-crystalline $\mathrm{Bi}_{2} \mathrm{Sr}_{2} \mathrm{CaCu}_{2} \mathrm{O}_{8+x}$ detectors for direct detection of microwave radiation
}

\author{
M. Li, ${ }^{\text {a) }}$ D. Winkler, and A. Yurgens \\ Department of Microtechnology and Nanoscience - MC2, Chalmers University of Technology, \\ SE-41296 Gothenburg, Sweden
}

(Received 3 February 2015; accepted 10 April 2015; published online 17 April 2015)

\begin{abstract}
We test radiation detectors made from single-crystalline $\mathrm{Bi}_{2} \mathrm{Sr}_{2} \mathrm{CaCu}_{2} \mathrm{O}_{8+x}$ flakes put on oxidized Si substrates. The 100-nm-thick flakes are lithographically patterned into $4 \times 12 \mu \mathrm{m}^{2}$ large rectangles embedded in thin-film log-spiral antennas. The $\mathrm{SiO}_{2}$ layer weakens the thermal link between the flakes and the bath. Two modes of radiation detection have been observed. For a bolometric type of sensors a responsivity of $\sim 300 \mathrm{~V} / \mathrm{W}$ and a noise equivalent power of $30 \mathrm{nW} / \sqrt{\mathrm{Hz}}$ has been deduced at $70 \mathrm{~K}$. Much more sensitive is the non-bolometric device showing characteristics similar to a Golay-type detector while being at least a thousand times faster. Making smaller (sub- $\mu \mathrm{m}$ ) structures is expected to significantly improve the performance of these devices and makes them very competitive among other microwave and terahertz detectors. (C) 2015 AIP Publishing LLC.

[http://dx.doi.org/10.1063/1.4918788]
\end{abstract}

Superconducting bolometers have been a subject of intense interest due to their potential application as detectors for infrared and terahertz radiation. ${ }^{1}$ Bolometers based on high transition temperature $\left(T_{c}\right)$ superconductors (HTS) are easier to cool $^{2,3}$ while still yielding high sensitivity at wavelengths above $20 \mu \mathrm{m}$. HTS thin films, such as $\mathrm{YBa}_{2} \mathrm{Cu}_{2} \mathrm{O}_{7-\delta}$ (YBCO), ${ }^{4,5}$ have been extensively studied for bolometric applications. One advantage of HTS bolometers is high resistivity of the material, making it easier to adjust the overall resistance closer to impedances of planar terahertz $(\mathrm{THz})$ antennas. HTS are best grown on single-crystalline substrates to achieve lattice match. The thermal conductivity of these substrates is usually high. This makes sensortemperature variations due to incoming radiation small. The sensitivity of these thin film bolometers was improved by fabrication of suspended micro-bridges or membranes as substrates. ${ }^{6,7}$ However, such devices are often subject to micro-cracking after thermal cycling and poor stability of characteristics. 8

Single-crystalline HTS $\mathrm{Bi}_{2} \mathrm{Sr}_{2} \mathrm{CaCu}_{2} \mathrm{O}_{8+x}$ (BSCCO) has large superconducting gap and quite sharp resistive transition at $T_{c} \approx 86 \mathrm{~K}$, similarly to YBCO. BSCCO can be cleaved and thinned further down to a few atomic layers, similarly to how graphene is obtained from graphite, ${ }^{9}$ while still preserving good superconducting properties. ${ }^{10,11} \mathrm{~A}$ small volume of the radiation-absorbing element is then expected to decrease the response time constant of the bolometer. Compared to YBCO thin films, BSCCO single crystals have better chemical stability in humid atmosphere. Finally, BSCCO flakes can be laid down onto largely any substrate including plastics which normally have very low thermal conductivity. This is expected to increase sensitivity of the detector. BSCCO can also be grown as an epitaxial thin film. This, again, demands a single-crystalline substrate with usually high thermal conductivity. Bolometers made from such thin films have shown quite low responsivity between 1.3 and

a)lime@chalmers.se
2.4 V/W and a noise equivalent power (NEP) of $\sim 10^{-8} \mathrm{~W} /$ $\sqrt{\mathrm{Hz}}$ at $77 \mathrm{~K} .{ }^{12}$

Here, we report on fabrication and properties of antenna-integrated radiation detectors made from BSCCO single-crystalline flakes laid on $\mathrm{SiO}_{2} / \mathrm{Si}$ substrates. The detectors are sensitive to microwave radiation in a wide temperature range up to room temperature. There are two distinct operational modes of the detectors. The most sensitive one is the non-bolometric device showing characteristics similar to a Golay-type detector while being a thousand times faster. By analyzing thermal balance in the bolometers, we conclude that the device sensitivity can be significantly improved by decreasing the lateral sizes of the absorber.

Initial BSCCO single crystals with $T_{c} \sim 90 \mathrm{~K}$ were grown by a traditional flux method. ${ }^{13}$ Thin flakes (Fig. 1(a)) with a thickness of a few hundred nanometers are obtained by mechanical exfoliation using sticky tape. The flakes are then laid onto an oxidized high-resistivity silicon substrate. The $\mathrm{SiO}_{2}$ thickness is $0.8 \mu \mathrm{m}$ for all samples in this study. Adhesion between the freshly cleaved BSCCO and clean surface of $\mathrm{SiO}_{2}$ is sufficient to hold the flakes during all subsequent lithography steps. There are usually many flakes of different thicknesses and irregular shapes that are attached to the substrate. The thickness of the flakes can be roughly estimated in an optical microscope by judging from their colors. Flakes of $\$ 100 \mathrm{~nm}$ thick are semitransparent giving rise to interference effects and consequently to different colors.

The fabrication process of our BSCCO bolometers is schematically illustrated in Fig. 1(b). A $50 \mathrm{~nm}$ thick Ag thin film is deposited onto the fresh BSCCO surface as a protection layer during the following lithography processing. The thickness of all flakes is then measured more accurately using the optical profilometer (Wyko NT1100). We then choose a suitable flake and protect it by a photoresist patch $120 \times 120 \mu \mathrm{m}^{2}$ large during wet etching the rest away in the $\sim 10 \%$ nitric acid (step 2). By photolithography patterning, thermal evaporation, and lift-off, everything but a small square in the middle of the flake is covered by the $100 / 5 \mathrm{~nm}$ 


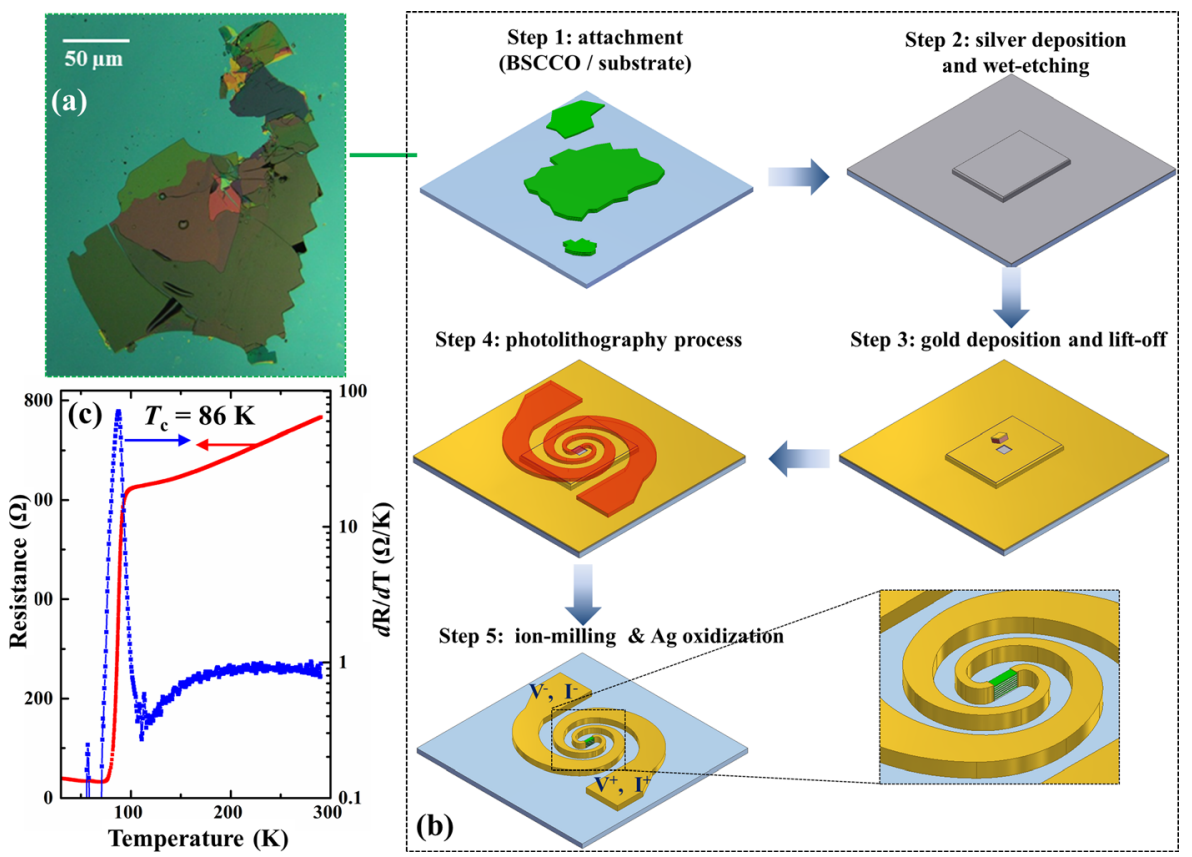

FIG. 1. (a) The optical image of a semi-transparent BSCCO flake adhered to $0.8 \mu \mathrm{m}$ thick $\mathrm{SiO}_{2}$ on top of a Si substrate. Different colors result from the light interference effect across the flake with spatially varying thickness. (b) Schematic view of the sample fabrication process. The dimensions are not to scale. (c) The inplane resistance $R(T)$ of the patterned BSCCO flake (red dots); calculated $d R / d T$ (blue dots). The inset shows the zoom-in of the superconductivity transition region.

$\mathrm{Au} / \mathrm{Cr}$ thin film (step 3). Finally, we pattern the photoresist etch mask having the shape of a log-spiral antenna crossing the square in the middle and etch through the whole flake by the Ar-ion milling (step 4). The photoresist is then removed by acetone and oxygen plasma. The Ag oxidation induced by the oxygen plasma is beneficial because it simultaneously removes the low-resistance shunt across the active part of the bolometer without additional Ar-ion etching (step 5). The sensing BSCCO part in the middle of the spiral antenna is $4 \times 12 \mu \mathrm{m}^{2}$ large and $\sim 0.1 \mu \mathrm{m}$ thick. The inner- and outerdiameters of the antenna are 36 and $510 \mu \mathrm{m}$, respectively, which roughly define the antenna bandwidth, $\sim 0.09-0.6$ THz. $^{14}$

The characteristics of the detectors are measured in a closed-cycle cryostat having optical (quartz) windows transparent for $\mathrm{THz}$ radiation. The samples are mounted on a hemispherical silicon lens with a diameter of $10 \mathrm{~mm}$ and placed in front of the cryostat windows. We normally use a Gunn-diode oscillator at $95 \mathrm{GHz}$ with a horn antenna and the maximum power $P_{0}=17 \mathrm{~mW}$. The radiation is modulated either by a mechanical chopper at $10-100 \mathrm{~Hz}$ or by using a pulsed bias of the Gunn oscillator $(\leq 10 \mathrm{kHz})$. Control experiments at $\mathrm{THz}$-range frequency were conducted by using a back wave oscillator (BWO) at $500 \mathrm{GHz}$. The voltage across the bolometer is amplified by an instrumentation amplifier $(\times 1-100)$ and synchronously measured by SR850 DSP (Digital Signal Processing) Lock-In Amplifier, usually using the $24-\mathrm{dB} /$ oct roll-off filter and $0.3 \mathrm{~s}$ of the time constant, which define the equivalent noise bandwidth of $0.14 \mathrm{~Hz}$.

Generally, a superconducting bolometer is heated up by both DC- and microwave power, which leads to a rise of the bolometer temperature and a change of its resistance. Then, at a constant bias current, the voltage change in response to microwaves is proportional to $d R / d T .^{15}$ As shown in Fig. 1 (c), $d R / d T$ for our bolometer is $\approx 70 \Omega / \mathrm{K}$ at $T=T_{c}$ and $\sim 1 \Omega / \mathrm{K}$ at room temperature. Compared with other HTS bolometers, the peak value of $d R / d T$ of our BSCCO bolometer is similar to that of the $\mathrm{GdBa}_{2} \mathrm{Cu}_{3} \mathrm{O}_{7-\delta}$ bolometer on silicon-nitride membrane or the $\mathrm{YBCO}$ bolometer on a bare silicon substrate. ${ }^{7,16}$

We present measurements on three devices with the same in-plane dimensions and similar thickness of $\sim 100 \mathrm{~nm}$. Two devices demonstrate a common bolometric behavior illustrated in Fig. 2. Typical current-voltage characteristics (IVCs) of the device $\mathrm{S} 1$ at $70 \mathrm{~K}$, with- and without-radiation, are shown in Fig. 2(a). In order to experimentally determine
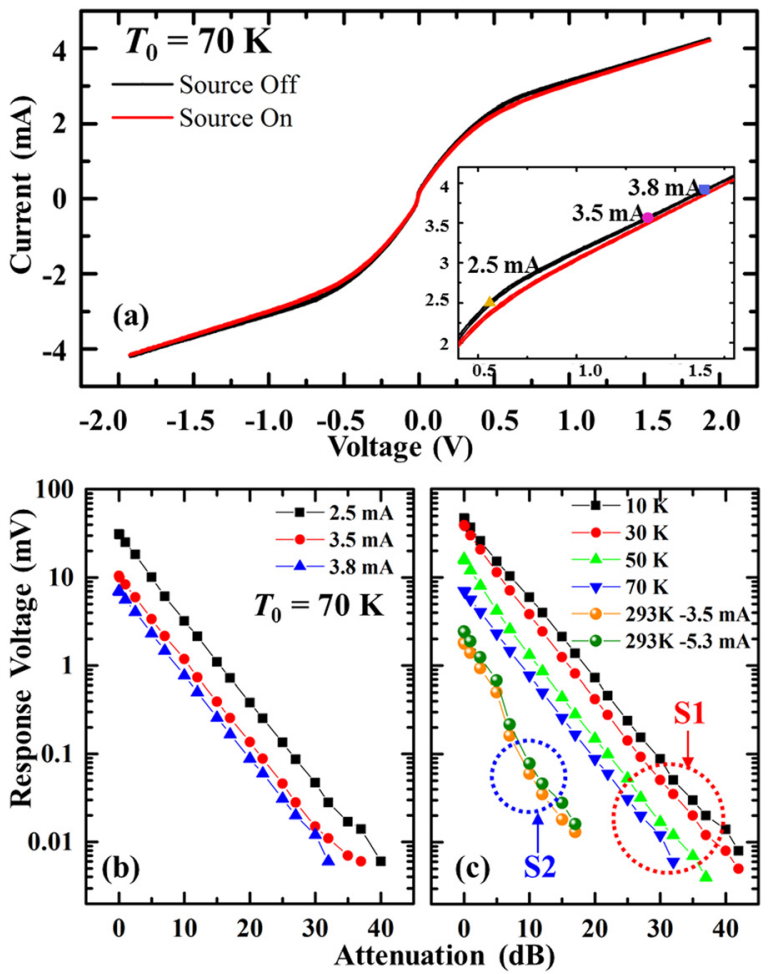

FIG. 2. (a) IVCs of sample $\mathrm{S} 1$ when the source is on (red) and off (black) at $70 \mathrm{~K}$. (b) Response rms voltage at $I=2.5,3.5$, and $3.8 \mathrm{~mA}$ at $70 \mathrm{~K}$ (see the inset of (a)) versus attenuation of the Gunn oscillator. (c) The response rms voltage at different bath temperatures versus attenuation for sample S1 at $I=3.8 \mathrm{~mA}$ and sample $\mathrm{S} 2$ at room temperature and $I=3.5$ and $5.3 \mathrm{~mA}$. 
sensitivity of the bolometers, we add an adjustable attenuator to the Gunn oscillator and measure the ac voltage resulting from the reduced radiation power modulated at $17 \mathrm{~Hz}$. We take the sensitivity of BSCCO bolometer as the minimum microwave power which is still detectable. Fig. 2(b) shows the response voltages at the bias currents of 2.5, 3.5, and $3.8 \mathrm{~mA}$ and at $T_{0}=70 \mathrm{~K}$. Generally, the response ac voltage exponentially decreases with the attenuation. The minimum detectable ac voltage for this sample is $\sim 10 \mu \mathrm{V}$ at these bias currents, mainly due to electrical noise from the compressor of our pulse-tube cryostat. With the compressor switched off, the noise voltage drops to below $1 \mu \mathrm{V}$, corresponding to the microwave power of $\sim 2 \mu \mathrm{W}$. Fig. 2(c) illustrates the response voltage at different $T_{0}$ at $I=3.8 \mathrm{~mA}$. The response voltage, in general, decreases with $T_{0}$.

The non-zero $d R / d T$ shown in Fig. 1(c) indicates that BSCCO bolometers can also work at room temperature although with reduced sensitivity. The IVCs at room temperature are show in Fig. 2(c). The response ac voltage also shows an exponential decrease with attenuation. The maximum response voltage is $V_{a c}=2.5 \mathrm{mV}$ at $I=5.3 \mathrm{~mA}$, which is nearly hundred times less than that at $10 \mathrm{~K}$ for the same sample. The minimum detectable power at room temperature is $\sim 20 \mu \mathrm{W}$, which is comparable with commercial room temperature $\mathrm{THz}$ detectors. ${ }^{17}$

Surprisingly, sample $\mathrm{S} 3$ revealed almost 100 times better sensitivity at low temperature. Fig. 3(a) shows that IVCs have horizontal parts which shift under the microwave radiation (see Fig. 3(b)). At a constant current, the shift yields a signal voltage, which only weakly depends on the dc voltage (see Fig. 3(c)) and is independent of the radiation-modulation frequency of up to $10 \mathrm{kHz}$. Note that it is only $50 \mu \mathrm{W}$ of microwave power which gives such a strong signal. We have also checked that the high responsivity of this detector holds even for $\mathrm{THz}$ radiation, by using a $\mathrm{BWO}$ at $\sim 500 \mathrm{GHz}$.

As will be clear from estimations below, the small dc power $(0.1 \mathrm{~mW})$ excludes the possibility of Joule heating the
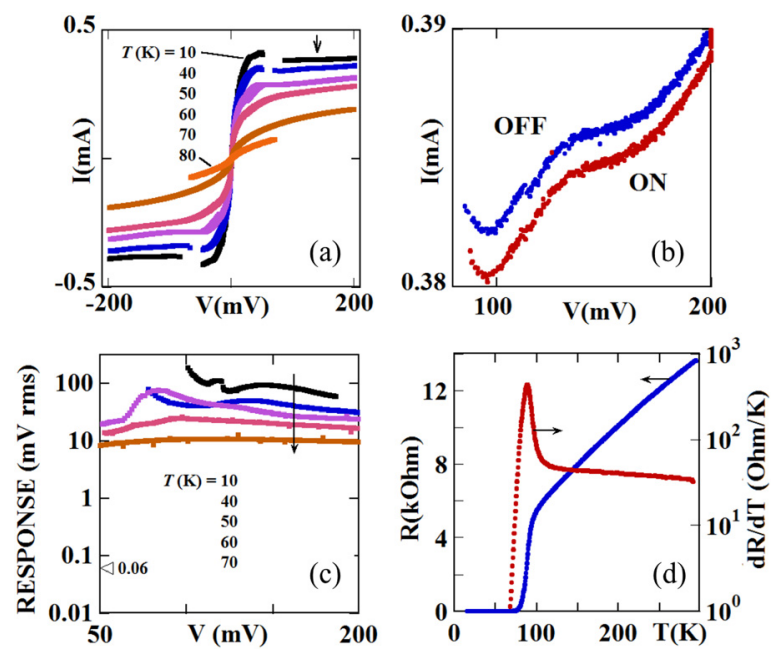

FIG. 3. (a) IVCs of sample S3 at different temperatures indicated. The arrow points to a nearly horizontal IVC part which is most sensitive to microwaves. (b) The zoomed-in part of IVC at $10 \mathrm{~K}$ upon switching on the radiation $(95 \mathrm{GHz}, \sim 50 \mu \mathrm{W})$. (c) The amplified $(\times 50)$ lock-in signal as a function of dc voltage across the sample at different temperatures. The triangle marks the noise level. (c) The temperature dependence of resistance $R$ and $d R / d T$. sample to above $T_{c}$ contrary to usual scenario for superconducting bolometers, in general. $R(T)$ is also smooth and featureless (see Fig. 3(d)). This calls for a different mechanism of the radiation detection which is, however, not clear at the moment. The current-driven resistive transition in a few superconducting layers at the surface of BSCCO single crystal might give a clue to the exact mechanism. ${ }^{18}$ More experiments are under way.

The electrothermal behavior of a bolometer can qualitatively be described by Newton's law of cooling: $T=T_{0}+I^{2} R(T) / G$, where $T$ is the temperature of the bolometer and $G$ is the overall conductance of the thermal link to the bath. It is usually assumed that $G$ is nearly constant which is justified for small heat dissipation and $T \sim T_{0}$. It is, however, difficult to apply this equation to get quantitative information for BSCCO bolometers. The temperature is anticipated to be spatially inhomogeneous and much higher than $T_{0}$. For instance, taking the thermal conductivity of $\mathrm{SiO}_{2}$ layer $\kappa_{q} \approx 1 \mathrm{~W} /(\mathrm{m} \cdot \mathrm{K})$, its thickness $t=0.8 \mu \mathrm{m}$, and the bolometer area of $4 \times 12=48 \mu \mathrm{m}^{2}$, we get $G=60 \mu \mathrm{W} / \mathrm{K}$ which for $R=600 \Omega$ gives $\left(T-T_{0}\right) \approx 160 \mathrm{~K}$ at $I=4 \mathrm{~mA}$.

For a more realistic quantitative analysis, we solve the coupled heat-diffusion- and electrical-transport equations by using the finite-element program Comsol. ${ }^{19}$ The spiral antenna is simplified to rectangular pads adjacent to the BSCCO flake also functioning as electrodes. Since the thickness of the flake is much smaller than the in-plane dimensions, the temperature gradient in the $c$-axis direction is not taken into account. Therefore, we assume the constant $c$-axis resistivity of $1 \Omega \mathrm{cm}$, the experimental in-plane resistivity as in Fig. 1(c), and the temperature-dependent thermal conductivity of $\mathrm{SiO}_{2}$ from Ref. 20. We numerically calculate the corresponding IVCs and compare them to the experimental data at $70 \mathrm{~K}$. Fig. 4 demonstrates that the simulations match the measurements quite well. It is seen that the temperature is highly inhomogeneous in the $a b$-plane (see the inset of Fig. 4) varying from 80 to $177 \mathrm{~K}$ at $I=3.5 \mathrm{~mA}$. The maximum $T$ exceeds $T_{c}$ at high bias current even for $T_{0}=10 \mathrm{~K}$.

We now calculate the responsivity $R_{v}$, thermal time constant $\tau$, and NEP for the bolometers. $R_{v}$ is the ratio of output voltage to the absorbed microwave power $R_{v}=V_{a c} / P$. To estimate $P$, we make several necessary assumptions. The transmittance through $N=3$ windows of our cryostat is $T r=(1-r) /(1+(2 N-1) r) \approx 0.5$, where $r=(n-1)^{2} /$ $(n+1)^{2} \approx 0.14$ is the reflection loss at one window surface

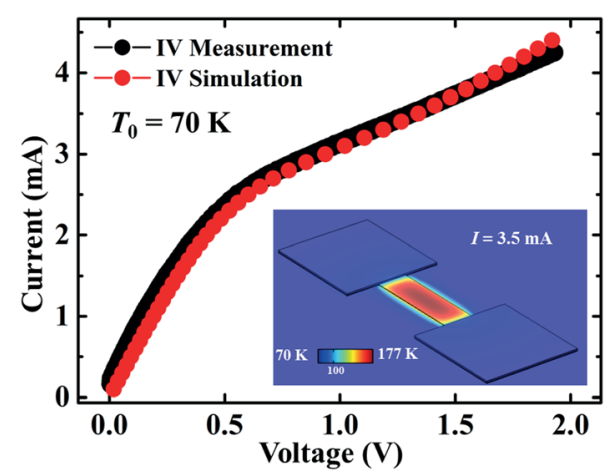

FIG. 4. Experimental IVC (black) and the simulated one (red) for a BSCCO bolometer at $T_{0}=70 \mathrm{~K}$. 
and $n=2.16$ is the index of refraction for quartz. ${ }^{21}$ Next, we take the reflection loss at the Si-lens surface 0.3 and the coupling factor to the spiral antenna of about 0.5 . We assume a common $21 \mathrm{~dB}$ gain of the horn antenna attached to the Gunn oscillator with the total power $P_{0}=17 \mathrm{~mW}$, which is $14 \mathrm{~cm}$ away from the detector Si lens. Putting everything together, $P \lesssim 0.009 P_{0} \approx 0.16 \mathrm{~mW}$ and $R_{v}$ is $\sim 320,44$, and $13 \mathrm{~V} / \mathrm{W}$ for the ac output signal of $50 \mathrm{mV}$ at $10 \mathrm{~K}, 7 \mathrm{mV}$ at $70 \mathrm{~K}$, and $2 \mathrm{mV}$ at room temperature, respectively (see Fig. 2(c)). NEP is defined as the incident signal power required to obtain a signal equal to the noise in $1 \mathrm{~Hz}$ bandwidth. ${ }^{1}$ Taking the noise voltage of $0.5 \mu \mathrm{V}$ and the equivalent noise bandwidth of $0.14 \mathrm{~Hz}, N E P$ can be estimated to be 4,30 , and $110 \mathrm{nW} / \sqrt{\mathrm{Hz}}$, respectively. For sample $\mathrm{S} 3$, there is an additional attenuation of $25.3 \mathrm{~dB}$ and noise $(15 \mu \mathrm{V})$ is higher because of $\times 50$ preamplification; $R_{v} \approx 2 \times 10^{5} \mathrm{~V} / \mathrm{W}$ and $N E P \approx 0.2 \mathrm{nW} / \sqrt{\mathrm{Hz}}$ can be obtained in a similar way. The latter values are very similar to the parameters of a Golay-cell detector. However, BSCCO detector promises to be much faster. Indeed, for BSCCO density $=6.5 \mathrm{~g} / \mathrm{cm}^{3}, 22$ the thermal time constant $\tau=C / G \approx 5$ ns can be estimated using specific heat of BSCCO absorber $c \approx 11 \mathrm{~J} /(\mathrm{kg} \cdot \mathrm{K})$ at $100 \mathrm{~K} .^{23}$ Experimentally, we tested the modulation frequency of only up to $10 \mathrm{kHz}$, with no observable change of the response signal.

BSCCO potentially allows for an ultimately small absorber and significantly improved characteristics. Indeed, BSCCO preserves its $T_{c}$ even for $d \sim 1$-nm thick single crystals. ${ }^{11}$ Reducing also lateral sizes to sub- $\mu \mathrm{m}$ range, the absorber volume can be made more than four orders of magnitude smaller making the devices about three orders of magnitude faster. This corresponds to frequencies reaching $20-40$ $\mathrm{GHz}$ allowing for wide-bandwidth mixing experiments. We note on passing that $G$ also decreases with decrease of the characteristic lateral size $a$ of the planar absorber on top of a thermally insulating layer of the thickness $t_{i}$. For $a<t_{i}$, the heat flow acquires a three-dimensional character for which $G \sim \kappa_{q} a{ }^{24}$ This means that for small enough, both the device sensitivity and its response time are improved.

In summary, characteristics of the single-crystalline BSCCO bolometers are competitive compared to other HTS detectors. Our devices perform well both at low- and room temperatures.

Financial support of the Swedish Research Council, the Swedish Foundation for Strategic Research, and the Knut and Alice Wallenberg Foundation was greatly appreciated. The samples were fabricated in the Nanofabrication Laboratory at MC2. The authors thank S. Cherednichenko for his advice, for his help with starting up our BWO, and for loaning us various mm-wave parts.

${ }^{1}$ P. Richards, J. Appl. Phys. 76, 1 (1994).

${ }^{2}$ P. Richards and L. Greenberg, Infrared Detectors for Low-Background Astronomy: Incoherent and Coherent Devices from One Micrometer to One Millimeter, Vol. 6 (ACADEMIC PRESS, INC., 1982), pp. 149-207.

${ }^{3}$ J. Clarke, G. Hoffer, P. Richards, and N.-H. Yeh, J. Appl. Phys. 48, 4865 (1977).

${ }^{4}$ A. Hammar, S. Cherednichenko, S. Bevilacqua, V. Drakinskiy, and J. Stake, IEEE Trans. Terahertz Sci. Technol. 1, 390 (2011).

${ }^{5}$ S. Cherednichenko, A. Hammar, S. Bevilacqua, V. Drakinskiy, J. Stake, and A. Kalabukhov, IEEE Trans. Terahertz Sci. Technol. 1, 395 (2011).

${ }^{6}$ P. Merel, P. Laou, and F. Wong, Proc. SPIE 5783, 607-615 (2005).

${ }^{7}$ M. De Nivelle, M. Bruijn, R. De Vries, J. Wijnbergen, P. De Korte, S. Sanchez, M. Elwenspoek, T. Heidenblut, B. Schwierzi, W. Michalke et al., J. Appl. Phys. 82, 4719 (1997).

${ }^{8}$ N. Khare, Handbook of High-Temperature Superconductor (CRC Press, 2003).

${ }^{9}$ K. S. Novoselov, A. K. Geim, S. Morozov, D. Jiang, Y. Zhang, S. Dubonos, I. Grigorieva, and A. Firsov, Science 306, 666 (2004).

${ }^{10}$ X. Wang, L. You, D. Liu, C. Lin, X. Xie, and M. Jiang, Physica C 474, 13 (2012).

${ }^{11}$ D. Jiang, T. Hu, L. You, Q. Li, A. Li, H. Wang, G. Mu, Hi. Chen, H. Zhang, G. Yu, J. Zhu, Q. Sun, C. Lin, H. Xiao, X. Xie, and M. Jiang, Nat. Commun. 5, 5708 (2014).

${ }^{12}$ A. Bottiglione, M. Epifani, G. Balestrino, E. Milani, M. Montuori, L. Maritato, A. Ruosi, and R. Scafuro, J. Appl. Phys. 74, 704 (1993).

${ }^{13}$ N. Zavaritsky, A. Samoilov, and A. Yurgens, Physica C 180, 417 (1991).

${ }^{14}$ A. D. Semenov, H. Richter, H.-W. Hubers, B. Gunther, A. Smirnov, K. S. Il'in, M. Siegel, and J. P. Karamarkovic, IEEE Trans. Microwave Theory Tech. 55, 239 (2007).

${ }^{15}$ A. J. Kreisler and A. Gaugue, Supercond. Sci. Technol. 13, 1235 (2000).

${ }^{16}$ D. G. McDonald, R. Phelan, Jr., L. R. Vale, R. H. Ono, and D. A. Rudman, IEEE Trans. Appl. Supercond. 9, 4471 (1999).

${ }^{17}$ See detailed at http://www.gentec-eo.com and http://www.mtinstruments.com for the specifics of commercial $\mathrm{THz}$ detectors.

${ }^{18}$ L. You, A. Yurgens, and D. Winkler, Phys. Rev. B 71, 224501 (2005).

${ }^{19}$ See http://www.comsol.com for the user's guide.

${ }^{20}$ R. Zeller and R. Pohl, Phys. Rev. B 4, 2029 (1971).

${ }^{21}$ R. A. Hull, Proc. Phys. Soc. 48, 574 (1936).

${ }^{22}$ T. Sugimoto, M. Yoshida, K. Sugawara, and Y. Shiohara, Advances in Superconductivity III (Springer, Japan, 1991), p. 1117.

${ }^{23}$ D. A. Cardwell and D. S. Ginley, Handbook of Superconducting Materials Volume 1: Superconductivity, Materials and Processes (IOP Publishing, 2003).

${ }^{24}$ V. Krasnov, A. Yurgens, D. Winkler, and P. Delsing, J. Appl. Phys. 89, 5578 (2001). 